\title{
Signatures of interaction-induced helical gaps in nanowire quantum point contacts
}

\author{
S. Heedt ${ }^{1}{ }^{\dagger}$, N. Traverso Ziani ${ }^{2}$, F. Crépin ${ }^{3}$, W. Prost ${ }^{4}$, St. Trellenkamp ${ }^{1}$, J. Schubert ${ }^{1}$, D. Grützmacher ${ }^{1}$, \\ B. Trauzettel ${ }^{2}$ and Th. Schäpers ${ }^{1 \star}$
}

Spin-momentum locking in a semiconductor device with strong spin-orbit coupling (SOC) is thought to be an important prerequisite for the formation of Majorana bound states ${ }^{1-3}$. Such a helical state is predicted in one-dimensional (1D) nanowires subject to strong Rashba SOC and spin-mixing ${ }^{4}$-its hallmark being a characteristic re-entrant behaviour in the conductance. Here, we report direct experimental observations of the re-entrant conductance feature, which reveals the formation of a helical liquid, in the lowest 1D subband of an InAs nanowire. Surprisingly, the feature is very prominent also in the absence of magnetic fields. This behaviour suggests that exchange interactions have a substantial impact on transport in our device. We attribute the opening of the pseudogap to spin-flipping two-particle backscattering ${ }^{5-7}$. The all-electric origin of the ideal helical transport could have important implications for topological quantum computing.

A 1D conductor with strong SOC is predicted ${ }^{1,2,8}$ to represent a viable host for Majorana bound states. These zero-energy states feature characteristic non-Abelian exchange statistics ${ }^{8}$ and can be created by mimicking spinless $p$-wave Cooper pairing using a semiconductor nanowire with a helical state and inducing $s$-wave superconductivity. InAs and InSb nanowires are promising host materials to explore the existence and nature of Majorana bound states $^{9,10}$. To this end, it is essential to both establish transport in $1 \mathrm{D}$ subbands and induce a helical state in the nanowire. The usual mechanism that is considered to open a helical gap involves an external Zeeman field oriented perpendicular to the uniaxial spinorbit field ${ }^{4}$. The magnitude of the spin-orbit energy relative to the Zeeman energy is partly responsible for the size of the topological energy gap that will protect the zero-energy Majorana modes ${ }^{11}$. However, Oreg et al. ${ }^{2,12}$ and Stoudenmire et al. ${ }^{13}$ have pointed out that such an energy gap can also result from strong electronic correlations. Several mechanisms have been proposed along these lines: for example, spin-flipping two-particle backscattering ${ }^{7}$ and hyperfine interaction between nuclear spins and a Luttinger liquid ${ }^{14}$, both of which can open a gap. The latter mechanism has been invoked to explain a conductance reduction by a factor of two at low temperatures in a GaAs quantum wire ${ }^{15}$, but no re-entrant behaviour is predicted within this framework.

Other than Quay et al. ${ }^{3}$, we report on a re-entrant conductance feature in the lowest subbands of InAs nanowire quantum point contacts (QPCs), which offer the desired strong SOC (see Supplementary Section 1). Moreover, our proposed spin-mixing mechanism does not necessarily rely on external time-reversal symmetry-breaking terms: while the effect is pronounced in the presence of an external magnetic field, it persists also in its absence. Guided by the observation ${ }^{16}$ of the Landé $g$ factor enhancement for the lowest subband ${ }^{17}$ and by signatures of the 0.7 anomaly ${ }^{18}$, we identify the important role of exchange interaction. Following ref. 7 , we ascribe the re-entrant behaviour at zero magnetic field to the combined presence of $1 \mathrm{D}$ confinement, Rashba spin-orbit coupling and Coulomb interaction.

The experimental set-up and the main results are summarized in Fig. 1. A scanning electron microscopy (SEM) image of the investigated device is depicted in Fig. 1a. The contact separation is $2.94 \mu \mathrm{m}$ and the wire diameter is $100 \mathrm{~nm}$. Top-gate fingers of $180 \mathrm{~nm}$ width can be used to form local QPCs in the nanowire. Enhanced gate coupling is enabled via a high- $k$ dielectric $\left(\mathrm{LaLuO}_{3}\right)$. The $\mathrm{Si} / \mathrm{SiO}_{2}$ back gate can induce an additional electric field in the QPCs. The conductance $G$ in Fig. $1 b$ is quantized in integer steps of $2 e^{2} / h$, while at large magnetic field $B$ half-integer steps emerge, reflecting the Zeeman splitting of the first subband, which is determined by a $g$ factor of $\sim 7.0^{16}$. A single re-entrant conductance feature appears reproducibly on the first quantized conductance plateau $\left(G=2 e^{2} / h\right)$ for magnetic fields smaller than $5 \mathrm{~T}$. It exhibits a non-monotonous behaviour, where the conductance drops by up to a factor of two and increases again to the integer quantized conductance value at higher energies, as depicted in Fig. $1 \mathrm{~b}$ and in the inset. The energy spectrum of the QPCs is reflected in the transconductance measurement as a function of the out-of-plane magnetic field. As shown in Fig. 1c, the subband edges rise in energy for larger magnetic fields and eventually converge towards Landau levels ${ }^{16,19}$. The riser at the right edge of the re-entrant conductance feature is related to the high-transconductance line marked by the green dot in Fig. 1c, which continuously evolves into the riser that separates the first two Zeeman-split plateaus at large magnetic fields. This behaviour is expected for a pseudogap that develops into a generic Zeeman splitting. At the single-particle level the physics is rather simple. Owing to the inversion asymmetry of the device, Rashba SOC gives rise to a spin-dependent shift of the subbands in momentum-space (Fig. 2a). For an electric field E perpendicular to the substrate plane, the spin-orbit field $\mathbf{B}_{\text {so }}$ is aligned perpendicular to the nanowire and in the plane of the substrate (see Fig. 2d, inset). The spinorbit energy $E_{\mathrm{so}}=m^{*} \alpha_{\mathrm{R}}^{2} / 2 \hbar^{2}$ can be calculated from the Rashba coefficient $\alpha_{\mathrm{R}}$ and it represents the energy difference between the degeneracy point at wave number $k=0$ and the band minima,

\footnotetext{
${ }^{1}$ Peter Grünberg Institut (PGI-9) and JARA-Fundamentals of Future Information Technology, Forschungszentrum Jülich, 52425 Jülich, Germany.

${ }^{2}$ Institute of Theoretical Physics and Astrophysics, University of Würzburg, 97074 Würzburg, Germany. ${ }^{3}$ Laboratoire de Physique Théorique de la Matière Condensée, UPMC, CNRS UMR 7600, Sorbonne Universités, 4 place Jussieu, 75252 Paris Cedex 05, France. ${ }^{4}$ Solid State Electronics Department, University of Duisburg-Essen, 47057 Duisburg, Germany. `Present address: QuTech, Delft University of Technology, 2628 CJ Delft, The Netherlands *e-mail: th.schaepers@fz-juelich.de
} 

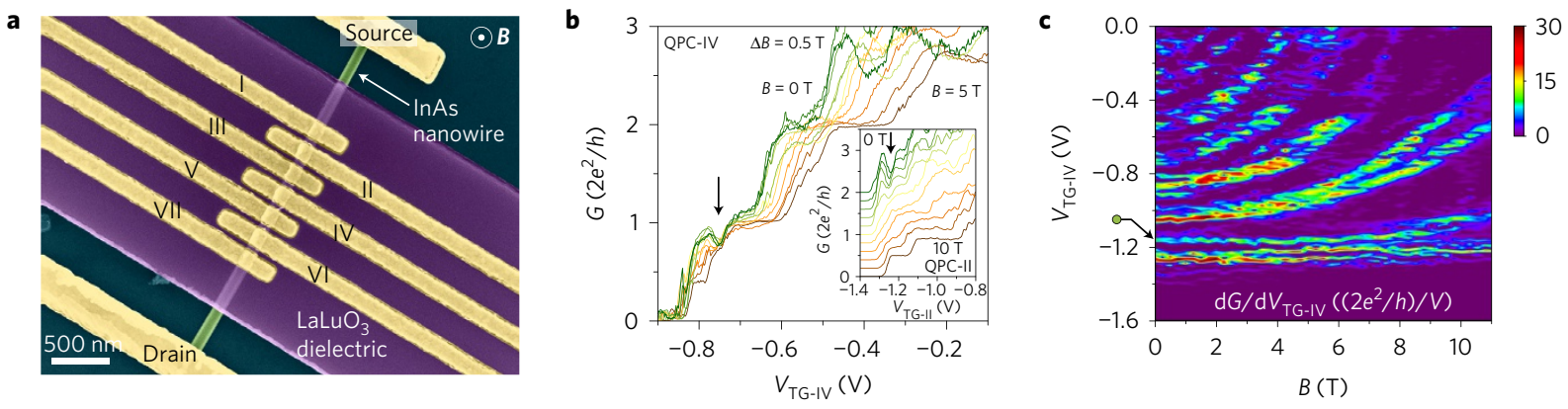

Figure 1 | Quantized conductance and the pseudogap feature. a, Top-view SEM image of the InAs nanowire covered with a layer of the high- $k$ dielectric $\mathrm{LaLuO}_{3}$. Each of the top-gate (TG) electrodes can be used to deplete the channel and control the number of $1 \mathrm{D}$ subbands. $\mathbf{b}$, Re-entrant conductance behaviour on the first plateau for QPC-IV (d.c.-bias voltage $V_{\text {d.c. }}=4 \mathrm{mV}$ ) at $T=100 \mathrm{mK}$ for various magnetic fields $B$. Inset: zero-bias conductance at $T=4 \mathrm{~K}$ for a different QPC (QPC-II) for $B$ increasing from 0 to $10 \mathrm{~T}$ ( $\Delta B=1 \mathrm{~T}$ ) with the re-entrant conductance feature on the $2 \mathrm{e}^{2} / \mathrm{h}$-plateau. Here, the back-gate voltage is fixed at $V_{\mathrm{BG}}=2 \mathrm{~V}$ and the curves are offset for clarity. Black arrows indicate the feature location. $\mathbf{c}$, $\mathrm{QPC}$-IV transconductance as a

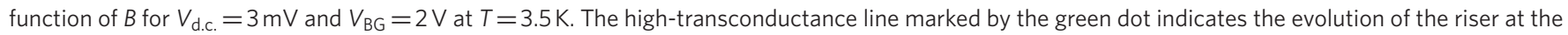
edge of the dip feature into the Zeeman-split subband edge at large $B$.

where $m^{*}$ is the effective electron mass (see Fig. $2 \mathrm{a}$ ). In the presence of a strong uniaxial spin-orbit field, a perpendicular magnetic field is expected to open a partial Zeeman gap, giving rise to quasi-helical transport for $E_{\mathrm{Z}}=g \mu_{\mathrm{B}} B \simeq E_{\mathrm{so}}{ }^{4}$ (see Fig. $2 \mathrm{~b}$ ). The formation of a helical state becomes manifest by the appearance of a re-entrant conductance plateau at $e^{2} / h$ inside the larger $2 e^{2} / h$-plateau related to the opening of the pseudogap (Fig. 2d, top panel). In our experiment, the gap widens roughly proportional to $g \mu_{\mathrm{B}} B$ (see Fig. 3). This aspect of the re-entrant feature is in accordance with the simple single-particle picture just described. Moreover, with increasing magnetic field, the gap evolves towards the generic Zeeman splitting of a spin-degenerate band (see Fig. 2c), which is reflected by the emergence of a plateau at a conductance of $e^{2} / h$ (Fig. 2d, bottom panel). However, the experimental observation of the pseudogap feature down to $B=0 \mathrm{~T}$ (see Fig. 2e) reveals the need to go beyond the single-particle picture. The effect we propose to cause the zero-field gap is the combination of Coulomb interaction and the breaking of axial spin symmetry, which can be induced, for instance, by the joint effect of spin-orbit coupling and quantum confinement ${ }^{20}$. Since spin is not a conserved quantity, the effective interaction term arising in this framework is correlated two-particle backscattering, which is resonant at $k=0$, similar to the singleparticle backscattering caused by the magnetic field. An estimation for the corresponding gap is (see Supplementary Section 2)

$$
\Delta_{\text {hel }}=\frac{m^{* 4}\left(\alpha_{\mathrm{R}} / \hbar\right)^{7} e^{2} d}{\hbar^{4} \omega_{0}^{3} \varepsilon_{0} \varepsilon_{\mathrm{r}} \sqrt{\hbar / m^{*} \omega_{0}}}
$$

Using $m^{*}=0.026 m_{\mathrm{e}}$ ( $m_{\mathrm{e}}$ being the free electron mass), the relative permittivity $\varepsilon_{\mathrm{r}}=15.15$, the confinement energy $\hbar \omega_{0}=7 \mathrm{meV}$ (ref. 16) and $\alpha_{\mathrm{R}}=1.2 \mathrm{eV} \AA$, which is discussed below, only the screening length $d$ remains unknown. Assuming $d=1 \mathrm{~nm}$, compatible with the upper bound for the electron density in the QPC segments, and considering the expected exchange-mediated renormalization of the gap by a factor of up to 3 (ref. 14), we estimate a pseudogap of the order of $\Delta_{\text {hel }} \approx 2.5 \mathrm{meV}$, which is compatible with the experimental findings. In fact, using the gate lever arm $\left(\approx 0.04 \mathrm{eV} \mathrm{V}^{-1}\right)$, we can estimate the energy width of the exchange-mediated gap of $\Delta_{\text {hel }} \approx 1.1 \mathrm{meV}$ at $T=100 \mathrm{mK}$ (for example, see Fig. 2e).

With regard to the estimate of $\alpha_{R}$, we observe weak antilocalization in the open, unconfined regime and an avoided crossing in the magnetic field evolution of spin states in quantum dots formed in this device. Both findings substantiate the sizeable SOC in the nanowire (see Supplementary Section 1). Accounting for the gate lever arm, we derive a spin-orbit energy on the order of $2.4 \mathrm{meV}$ from the gate voltage position of the centre of the re-entrant conductance region (see Fig. $2 \mathrm{e}$ ). Thus, $\alpha_{\mathrm{R}} \approx 1.2 \mathrm{eV} \AA$, which is a factor of four larger than $\alpha_{\mathrm{R}}$ derived with conventional methods (see Supplementary Section 1). It is, however, of similar magnitude to the Rashba parameters found in InSb nanowires via weak antilocalization, which go up to $1 \mathrm{eV} \AA$ (ref. 21). It should be noted that those methods probably underestimate $E_{\text {so }}$, since they consider spin relaxation only in the weakly confined multimode regime.

Despite the conceptual simplicity, the visibility of the re-entrant behaviour in the conductance for any value of the magnetic field is not to be taken for granted: the unambiguous identification of the SOC-induced conductance feature is generally obstructed by the non-optimal gate potential shape forming the $\mathrm{QPC}^{22}$ (see Supplementary Section 3). Moreover, the helical gap can be obscured by Fabry-Pérot resonances that are superimposed on the quantized zero-bias conductance plateaus at low temperatures ${ }^{16,23}$. Also the shape of the constriction ${ }^{24}$ as well as local potential fluctuations $s^{25}$ can have a critical impact on the transmission. However, at $T>6 \mathrm{~K}$ the feature can still be observed (see Fig. 3a), while Fabry-Pérot oscillations largely disappear in this regime. This rules out phase-coherent interference as the origin of the effect. At high temperatures $(T \approx 9 \mathrm{~K})$ the re-entrant conductance feature is less pronounced, but it broadens with increasing magnetic field and evolves into the first $e^{2} / h$-plateau for $B>4 \mathrm{~T}$ (see Fig. $3 \mathrm{~b}$ ). Another process that could induce a re-entrant behaviour is reflection by impurities. Similar re-entrant conductance features have, however, been observed for all investigated QPCs. The reproducibility of the feature position for different QPCs indicates that resonant reflections due to backscattering at impurities in the constrictions ${ }^{26}$ are unlikely to explain the observed effect. An additional consistency check is provided by the fact that the re-entrant conductance behaviour appears as long as the Zeeman energy is of the order of or smaller than $E_{\mathrm{so}}$, which is a requirement for the existence of the helical gap.

Further validation of the helical nature of the re-entrant behaviour is provided by the analysis of the conductance as a function of Rashba SOC. The magnitude of spin-orbit coupling in the QPC is determined by the strong electric field from the top gate that creates the confinement potential of the constriction. A positive back-gate voltage $V_{\mathrm{BG}}$ does not only increase the conductance of the system, it also enhances the Rashba coefficient at the QPC ${ }^{21}$. As depicted in Fig. 4 , at $B=1.5 \mathrm{~T}$ the conductance dip is a welldefined singular feature at $V_{\mathrm{BG}} \gg 0$. It can be seen that for decreasing $V_{\mathrm{BG}}$ the feature becomes less pronounced, and finally develops into a double-plateau, which is characteristic of conventional Zeeman 

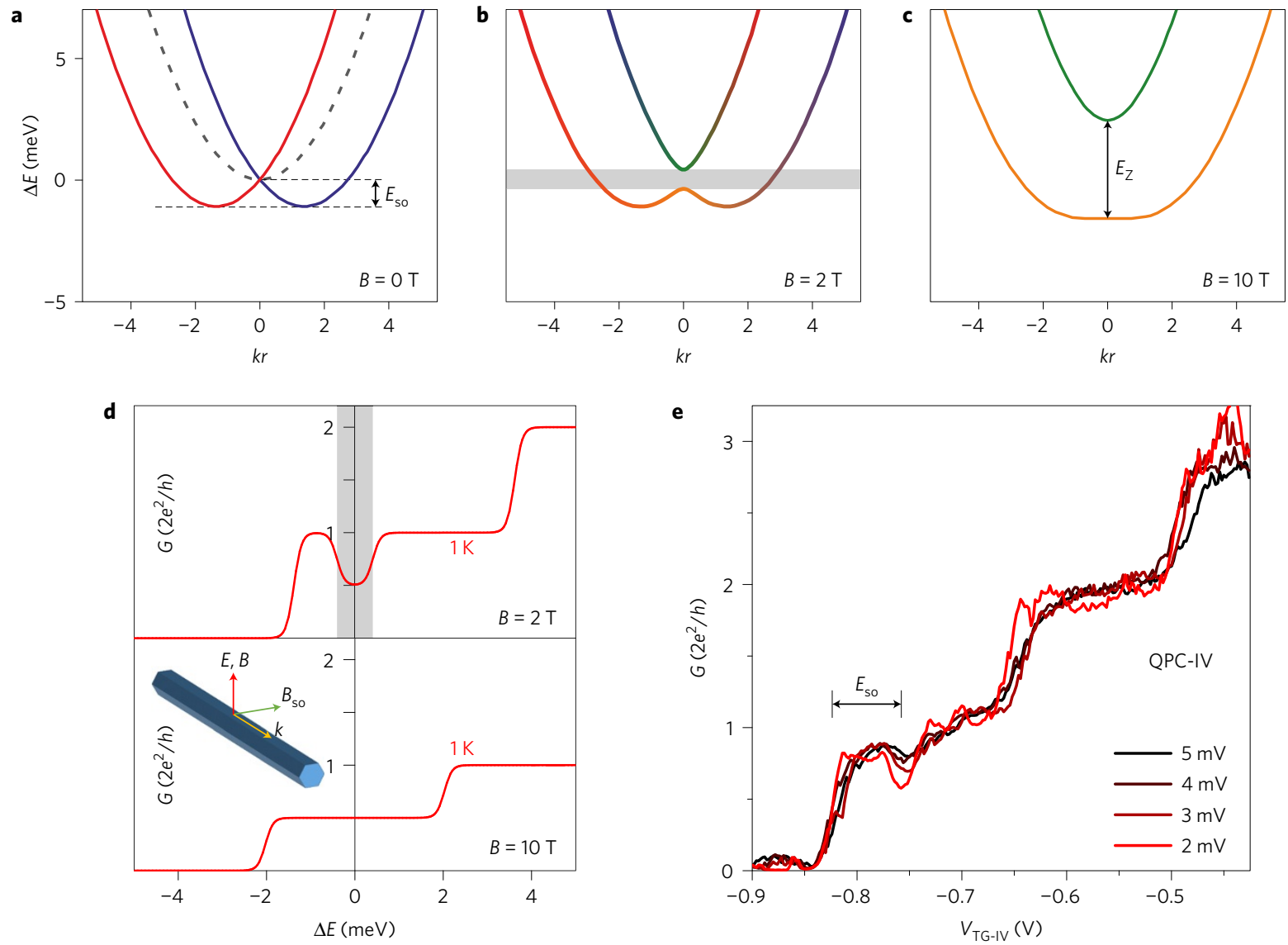

Figure 2 | Helical energy dispersion and bias-dependent re-entrant conductance feature. a-c, Dispersion relations for $\alpha_{\mathrm{R}}=0.8 \mathrm{eV} \AA$ at different magnetic fields $B$ (nanowire radius $r=50 \mathrm{~nm}$ ). $\mathbf{a}$, The two spinful subbands are lowered in energy by $E_{\mathrm{so}}=m^{*} \alpha_{\mathrm{R}}^{2} / 2 \hbar^{2}$ and shifted in $k$-space by $\pm m^{*} \alpha_{\mathrm{R}} / \hbar^{2}(B=0 \mathrm{~T}$ ). b. At $B=2 T$ the two spin bands are mixed by the magnetic field, which is perpendicular to the spin-orbit field $B_{\mathrm{so}}$, leading to an avoided crossing at $k=0$. Red and blue denote spin orientations along the spin-orbit field direction, while green and orange denote spins along the magnetic field direction. c, At $B=10 \mathrm{~T}$ the Zeeman energy dominates over $E_{\mathrm{so}}$. $\mathbf{d}$, Calculated conductance for the conditions in $\mathbf{b}$ (upper panel) and $\mathbf{c}$ (lower panel). e, Re-entrant feature on the first d.c. conductance plateau for QPC-IV $(T=100 \mathrm{mK})$ as a function of the d.c.-bias voltage at $B=0 \mathrm{~T}$.
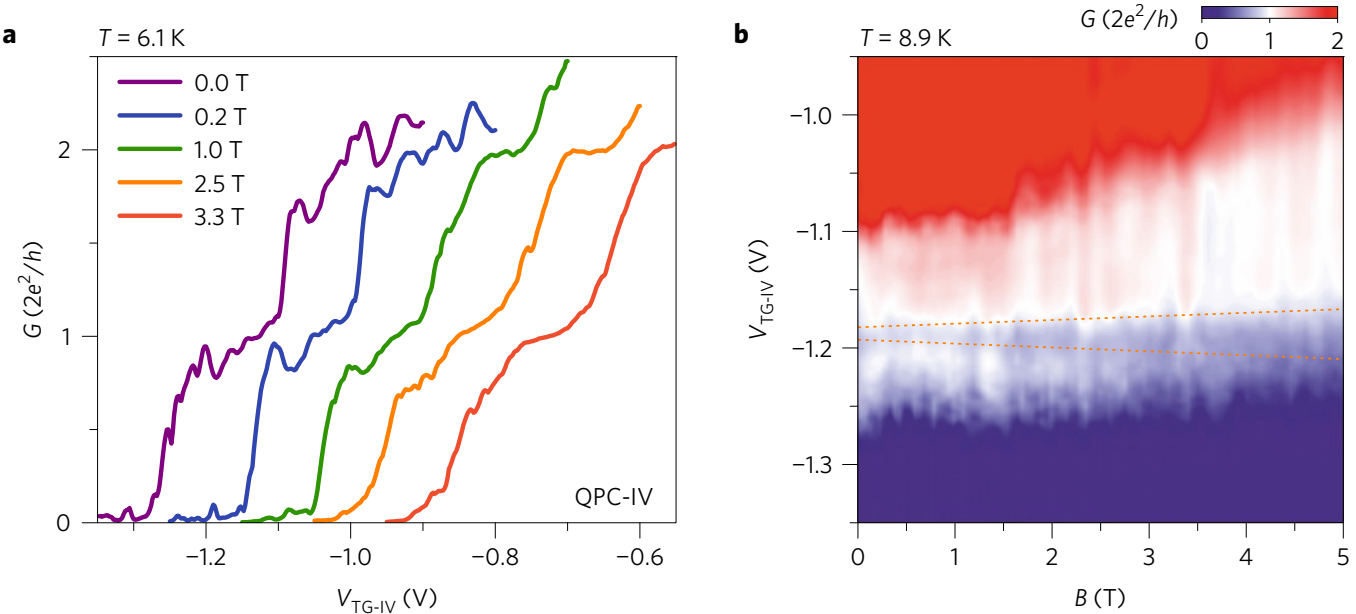

Figure 3 | Re-entrant conductance feature at higher temperatures. Conductance for QPC-IV at $T=6.1 \mathrm{~K}$ (offset in $V_{\mathrm{TG}-\mathrm{IV}}$ ) (a) and $T=8.9 \mathrm{~K}(\mathbf{b})$ for different magnetic fields. With increasing magnetic field, the dip feature on the first quantized conductance plateau broadens and the shoulder below the dip disappears at larger $B$. For $B>4 T$ the Zeeman energy contribution dominates over the spin-orbit energy.

splitting. The modified SOC strength is expected to manifest in a changed visibility of the re-entrant conductance feature. For $E_{\mathrm{so}} \lesssim g \mu_{\mathrm{B}} B+\Delta_{\text {hel }}$ the shoulder below the dip disappears and a single $e^{2} / h$-plateau remains.
In summary, a robust re-entrant conductance feature at the $2 e^{2} / h$-plateau is observed for all investigated QPCs along the nanowire. The variation with magnetic field, bias voltage, temperature and back-gate voltage reproduces the signatures of a helical 

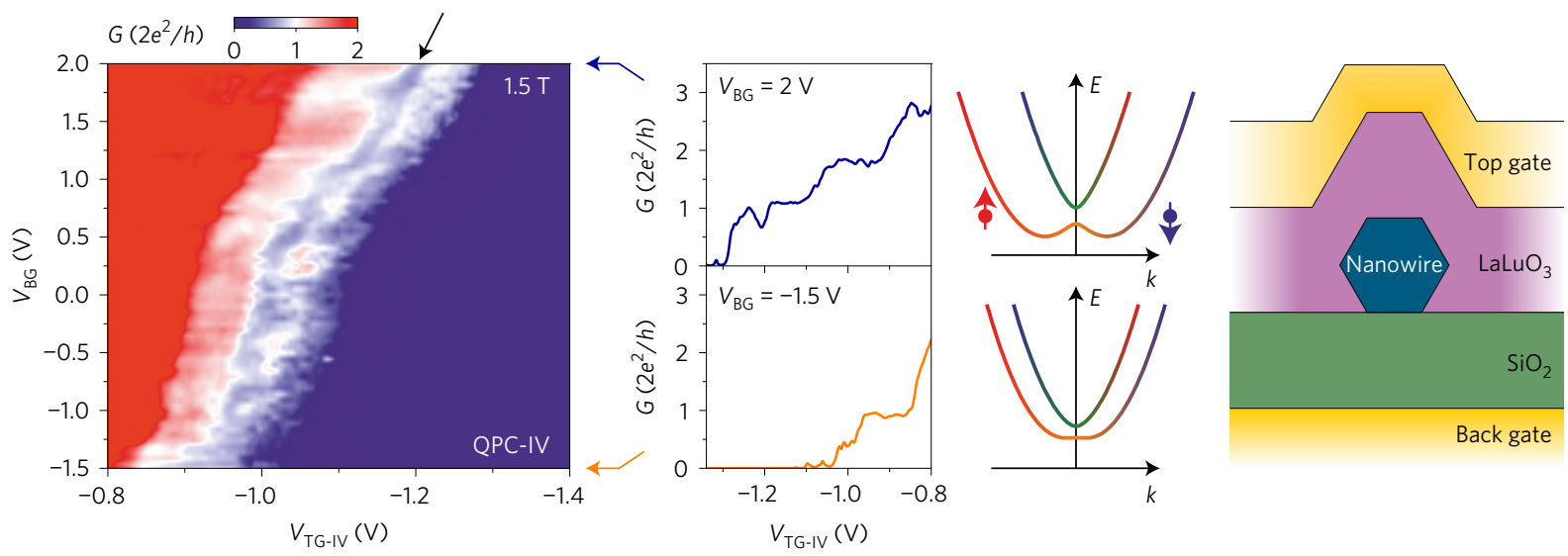

Figure 4 | Impact of the back-gate voltage on the re-entrant conductance feature. Conductance for QPC-IV at $T=4 \mathrm{~K}$ and $B=1.5 \mathrm{~T}$ as a function of the back-gate voltage. $V_{B G}$ changes the Fermi level and also tunes Rashba SOC. The black arrow points at the re-entrant feature. The characteristic re-entrant behaviour is most pronounced for $V_{B G} \gg 0$, as can be seen in the blue line cut for $V_{B G}=2 \mathrm{~V}$. The corresponding energy dispersion is illustrated to the right of the line cut. The conductance shoulder below the dip feature shrinks for decreasing $V_{B G}$. For $V_{B G} \ll 0$ the feature disappears and a double-step develops, as depicted in the orange line cut for $V_{\mathrm{BG}}=-1.5 \mathrm{~V}$ in agreement with the energy dispersion where $E_{\mathrm{so}} \lesssim E_{\mathrm{Z}}+\Delta_{\text {hel }}$. Right: cross-section of the dual-gate device.

gap. We suggest a spin-mixing mechanism based on strong exchange interaction to explain the gap opening at zero magnetic field. The observed spin-orbit energy of $2.4 \mathrm{meV}$ is attributed to a strong electric field that generates the distinct confinement in the QPC and enhances Rashba SOC. The all-electric nature of the helical gap preserves time-reversal symmetry and, in the presence of an induced superconducting gap, offers the desired conditions for fractional excitations that give rise to parafermionic quasiparticles ${ }^{27}$, which are promising building blocks in the context of topologically protected quantum computing ${ }^{28}$.

\section{Methods}

Methods, including statements of data availability and any associated accession codes and references, are available in the online version of this paper.

\section{Received 19 September 2016; accepted 14 February 2017;} published online 20 March 2017

\section{References}

1. Lutchyn, R. M., Sau, J. D. \& Das Sarma, S. Majorana fermions and a topological phase transition in semiconductor-superconductor heterostructures. Phys. Rev. Lett. 105, 077001 (2010).

2. Oreg, Y., Refael, G. \& von Oppen, F. Helical liquids and Majorana bound states in quantum wires. Phys. Rev. Lett. 105, 177002 (2010).

3. Quay, C. H. L. et al. Observation of a one-dimensional spin-orbit gap in a quantum wire. Nat. Phys. 6, 336-339 (2010).

4. Středa, P. \& Šeba, P. Antisymmetric spin filtering in one-dimensional electron systems with uniform spin-orbit coupling. Phys. Rev. Lett. 90, 256601 (2003).

5. Wu, C., Bernevig, B. A. \& Zhang, S.-C. Helical liquid and the edge of quantum spin Hall systems. Phys. Rev. Lett. 96, 106401 (2006).

6. Xu, C. \& Moore, J. E. Stability of the quantum spin Hall effect: effects of interactions, disorder, and $\mathbb{Z}_{2}$ topology. Phys. Rev. B 73, 045322 (2006).

7. Pedder, C. J., Meng, T., Tiwari, R. P. \& Schmidt, T. L. Dynamic response functions and helical gaps in interacting Rashba nanowires with and without magnetic fields. Phys. Rev. B 94, 245414 (2016).

8. Alicea, J., Oreg, Y., Refael, G., von Oppen, F. \& Fisher, M. P. A. Non-Abelian statistics and topological quantum information processing in $1 \mathrm{D}$ wire networks. Nat. Phys. 7, 412-417 (2011).

9. Mourik, V. et al. Signatures of Majorana fermions in hybrid superconductor-semiconductor nanowire devices. Science $\mathbf{3 3 6}$ 1003-1007 (2012).

10. Das, A. et al. Zero-bias peaks and splitting in an Al-InAs nanowire topological superconductor as a signature of Majorana fermions. Nat. Phys. 8 , 887-895 (2012).
11. Sau, J. D., Tewari, S. \& Das Sarma, S. Experimental and materials considerations for the topological superconducting state in electron- and hole-doped semiconductors: searching for non-Abelian Majorana modes in 1D nanowires and 2D heterostructures. Phys. Rev. B 85, 064512 (2012).

12. Oreg, Y., Sela, E. \& Stern, A. Fractional helical liquids in quantum wires. Phys. Rev. B 89, 115402 (2014).

13. Stoudenmire, E. M., Alicea, J., Starykh, O. A. \& Fisher, M. P. A. Interaction effects in topological superconducting wires supporting Majorana fermions. Phys. Rev. B 84, 014503 (2011)

14. Braunecker, B., Simon, P. \& Loss, D. Nuclear magnetism and electron order in interacting one-dimensional conductors. Phys. Rev. B 80, 165119 (2009).

15. Scheller, C. P. et al. Possible evidence for helical nuclear spin order in GaAs quantum wires. Phys. Rev. Lett. 112, 066801 (2014).

16. Heedt, S., Prost, W., Schubert, J., Grützmacher, D. \& Schäpers, Th. Ballistic transport and exchange interaction in InAs nanowire quantum point contacts. Nano Lett. 16, 3116-3123 (2016).

17. Martin, T. P. et al. Enhanced Zeeman splitting in $\mathrm{Ga}_{0.25} \mathrm{In}_{0.75}$ As quantum point contacts. Appl. Phys. Lett. 93, 012105 (2008).

18. Micolich, A. P. What lurks below the last plateau: experimental studies of the $0.7 \times 2 e^{2} / h$ conductance anomaly in one-dimensional systems. J. Phys. Condens. Matter 23, 443201 (2011).

19. Heedt, S. et al. Adiabatic edge channel transport in a nanowire quantum point contact register. Nano Lett. 16, 4569-4575 (2016).

20. Governale, M. \& Zülicke, U. Spin accumulation in quantum wires with strong Rashba spin-orbit coupling. Phys. Rev. B 66, 073311 (2002).

21. Van Weperen, I. et al. Spin-orbit interaction in InSb nanowires. Phys. Rev. B 91, 201413 (2015)

22. Rainis, D. \& Loss, D. Conductance behavior in nanowires with spin-orbit interaction: a numerical study. Phys. Rev. B 90, 235415 (2014).

23. Cayao, J., Prada, E., San-Jose, P. \& Aguado, R. SNS junctions in nanowires with spin-orbit coupling: Role of confinement and helicity on the subgap spectrum. Phys. Rev. B 91, 024514 (2015).

24. Tekman, E. \& Ciraci, S. Novel features of quantum conduction in a constriction. Phys. Rev. B 39, 8772-8775 (1989).

25. Nixon, J. A., Davies, J. H. \& Baranger, H. U. Breakdown of quantized conductance in point contacts calculated using realistic potentials. Phys. Rev. B 43, 12638-12641 (1991)

26. McEuen, P. L., Alphenaar, B. W., Wheeler, R. G. \& Sacks, R. N. Resonant transport effects due to an impurity in a narrow constriction. Surf. Sci. 229, 312-315 (1990)

27. Pedder, C. J., Meng, T., Tiwari, R. P. \& Schmidt, T. L. $\mathbb{Z}_{4}$ parafermions \& the $8 \pi$-periodic Josephson effect in interacting Rashba nanowires. Preprint at http://arxiv.org/abs/1507.08881 (2015).

28. Hutter, A. \& Loss, D. Quantum computing with parafermions. Phys. Rev. B 93 125105 (2016)

\section{Acknowledgements}

We gratefully acknowledge C. J. Pedder, T. Meng and T. L. Schmidt for fruitful discussions and H. Kertz for valuable help during the measurements. This work was 
supported by funding from the DFG (SPP1666 and SFB1170 ToCoTronics) and by the Virtual Institute for Topological Insulators (VITI), which is funded by the Helmholtz Association.

\section{Author contributions}

S.H., St.T. and J.S. fabricated the sample. S.H. performed the measurements. W.P. grew the InAs nanowires. N.T.Z., F.C. and B.T. derived the anomalous interaction term. S.H., Th.S., N.T.Z., F.C. and B.T. discussed the data. All authors contributed to the manuscript.

\section{Additional information}

Supplementary information is available in the online version of the paper. Reprints and permissions information is available online at www.nature.com/reprints. Publisher's note: Springer Nature remains neutral with regard to jurisdictional claims in published maps and institutional affiliations. Correspondence and requests for materials should be addressed to Th.S.

\section{Competing financial interests}

The authors declare no competing financial interests. 


\section{Methods}

Device fabrication. InAs nanowires are grown via gold-catalysed metal-organic vapour phase epitaxy on GaAs (111)B substrates ${ }^{29}$. The field-effect mobility was found to be $25,000 \mathrm{~cm}^{2} \mathrm{Vs}^{-1}$ (ref. 16) and the electron concentration is about $1.0 \times 10^{17} \mathrm{~cm}^{-3}$ (ref. 19). Nanowires are mechanically transferred onto a $\mathrm{Si}$ substrate with a 200 -nm-thick $\mathrm{SiO}_{2}$ layer that enables back-gate functionality. $\mathrm{LaLuO}_{3}$ dielectric ${ }^{30}$ is deposited onto the nanowire via pulsed laser deposition at room temperature and using a lift-off technique. The $\mathrm{LaLuO}_{3}$ layer is $100 \mathrm{~nm}$ thick and the relative permittivity is $\varepsilon_{\mathrm{r}}=26.9$. Ti/Au top-gate electrodes with $180 \mathrm{~nm}$ width and $30 \mathrm{~nm}$ pitch are fabricated. Following in situ $\mathrm{Ar}^{+}$sputtering of the nanowire, $120 \mathrm{~nm}$ of $\mathrm{Ti} / \mathrm{Au}$ electrodes are evaporated.

Measurements. All measurements have been carried out in a ${ }^{3} \mathrm{He} /{ }^{4} \mathrm{He}$ dilution refrigerator with a superconducting single-axis magnet. All conductance measurements at zero d.c.-bias voltage are performed at an a.c. excitation voltage of $V_{\text {a.c. }}=80 \mu \mathrm{V}_{\text {rms }}$. The bias voltage is applied symmetrically with respect to ground from source to drain electrode and the current is measured simultaneously at both ends of the nanowire. The actual voltage across the QPCs is significantly smaller than the applied voltage, considering the voltage drop across the series resistance related to non-ballistic nanowire segments. Typical values of the subtracted series resistance including contributions from the measurement set-up are of the order of $20 \mathrm{k} \Omega$, chosen such that the conductance plateaus match with integer multiples of $2 e^{2} / h$.

Model. The re-entrant feature in the absence of applied magnetic fields is explained by means of a Luttinger liquid model in which an electron-electron interaction term that couples the different spin species is introduced. The relevant process is dubbed correlated two-particle backscattering. It emerges as an effective interaction process due to the hybridization of different confinement subbands of the nanowire. We identify Rashba spin-orbit coupling as the most plausible physical mechanism responsible for the hybridization. This model is rather distinct from the description of the system as a (zig-zag) Wigner molecule ${ }^{31}$, which could be an alternative explanation for a variation of the conductance as a function of gate voltage. As we explain in Supplementary Section 2, the experimental signatures that we would expect from the formation of a Wigner molecule are incompatible with the observed features. We also discuss in Supplementary Section 2 that our model and its observable consequences are distinct from the 0.7 anomaly.

Data availability. The data that support the plots within this paper and other findings of this study are available from the corresponding author upon reasonable request.

\section{References}

29. Sladek, K. et al. Comparison of InAs nanowire conductivity: influence of growth method and structure. Phys. Status Solidi C 9, 230-234 (2012).

30. Durgun Özben, E. et al. Integration of $\mathrm{LaLuO}_{3}(\kappa \sim 30)$ as high- $\kappa$ dielectric on strained and unstrained SOI MOSFETs with a replacement gate process. IEEE Electron Device Lett. 32, 15-17 (2011).

31. Meyer, J. S. \& Matveev, K. A. Wigner crystal physics in quantum wires. J. Phys. Condens. Matter 21, 023203 (2009). 